\title{
Image Retrieval using Chain code and Autoregression
}

\author{
Salunkhe Shweta S. \\ Research Scholar, \\ Department of Electronics Engineering, \\ College of Engineering, \\ Bharati Vidyapeeth Deemed University, Pune, \\ Maharashtra, India
}

\author{
Gengaje Sachin R., PhD \\ Professor, \\ Department of Electronics Engineering, \\ College of Engineering, \\ Bharati Vidyapeeth Deemed University, Pune, \\ Maharashtra, India
}

\begin{abstract}
Over the years passed has witnessed great interest in research on content-based image retrieval. This has paved the way for a large number of new techniques and systems, and a growing interest in associated fields to support such systems. Similarly, digital image retrieval has expanded in many directions that are resulting into explosion in the volume of image data required to be organized. This paper presents a framework for image retrieval based on chain code and auto regression that helps to achieve higher retrieval efficiency. In this paper, we discuss about the key contributions of the methodology that is followed while performing experiment for image retrieval based on chain code and auto regression. Here comparative study of results and also efficiency of both these image retrieval techniques are discussed which are obtained while experimentation.
\end{abstract}

\section{Keywords}

Auto-regression, Chain code, Image retrieval

\section{INTRODUCTION}

Any image or an object we considered consists of many features which are encoded in it. That's why it becomes somewhat challenging to retrieve the image. Some techniques developed over the years are found very helpful in the image retrieval. These technologies may helps to develop automatic image retrieval systems that allow the image retrieving in real time. Image retrieval based on chain code and autoregression has a wide area of research scope. Auto regression is mathematical matrix calculation that calculates features using neighbourhood information of any pixel and chain code is contour based histogram calculation suited for shape recognition. In this experiment, we have discussed the performance of both these feature extraction techniques helpful for shape as well as image characteristic recognition.

Paper is organized as: Section II gives a brief overview of state of art methods under literature survey. Methodology of the proposed system is given in Section III. Section IV presents experimental results showing results under experimentation. Finally, Section V presents conclusions.

The experimental results demonstrate the efficacy of the method.

\section{LITERATURE SURVEY}

Utpal Garain, et.al [1] introduced a method of writer identification in a multiscript environment is attempted using a two dimensional (2D) autoregressive (AR) modelling technique. Each writer is represented by a set of $2 \mathrm{D}$ AR model coefficients. A method to estimate AR model coefficients is proposed. This method is applied to an image of text written by a specific writer so that AR coefficients are obtained to characterize the writer. For a given sample, AR coefficients are computed and its L2 distance with each of the stored (writer) prototypes identifies the writer for the sample. The method has been tested on datasets of two different scripts, namely RIMES (382 French writers) and ISI (40 Bengali writers). Modelling of writing styles using different context patterns at different image resolution has been investigated. Experimental results show that the technique achieves results comparable with that of the previous approaches.

Bandera, et.al [2] proposed a real time 2D object recognition algorithm. Contours are represented by their curvature functions, decomposed in the Fourier domain as linear combination of a set of representative objects. Finally, objects are identified by multilevel clustering.

Imran Siddiqi, et.al. [3] proposed an effective technique for writer recognition in handwritten documents. Here a set of features extracted from the contours of handwritten images at different observation levels are considered. At the global level, the histograms of the chain code are extracted, the first and second order differential chain codes and, the histogram of the curvature indices at each point of the contour of handwriting. At the local level, the handwritten text is divided into a large number of small adaptive windows and within each window the contribution of each of the eight directions (and their differentials) is counted in the corresponding histograms. Comparison of two writings is done by computing the distances between their respective histograms. The system trained and tested on two different data sets of 650 and 225 writers respectively.

Farida Khursheed, et.al [6] proposed a method on usefulness of time series based Auto Regressive (AR) modelling technique has been explored for identification of a person. For this purpose, time series is obtained from the contour coordinates of the ear. AR model is fitted to this time series. AR coefficients thus obtained serve as a feature vector. Recognition Rate (RR) has been found by a classifier that is based on Euclidian distance between feature vector of test samples with training samples within itself (intraclass) and with respect to others (interclass). Model has been found invariant to posture, rotation and illumination. RR up to $99 \%$ has been obtained. Results have been compared with existing techniques.

\section{METHODOLOGY}

Image retrieval technique initiates with the preprocessing. It involves image acquisition, gray conversion. Important and core part involves the feature extraction which can be done with the help of autoregression and chain code. In autoregression feature extraction completely depends upon mathematical modelling for shape detection whereas in case of chain code contour based feature extraction can be done. Methodology can be well understood as: 
A. Methodology based on Two-dimensional autoregressive model :

An image can be defined as discrete when $\mathrm{M} \times \mathrm{N}($ say, $P=$ $\mathrm{M} \times \mathrm{N}$ ) rectangular grid is denoted by

$$
\{x i j\}(i=1,2, \ldots, M ; j=1,2, \ldots, N) .
$$

Here every element $x i j$ is a random $\square \square$ variable. $\{x i j\}$ is called as discrete random field. Here, random linear equation can be given as

$$
x_{i j}=\sum_{(p, q \in D)} \theta_{p q} x_{i-p}, j-q
$$

here $D$ is the context region normally represented by a rectangular region as

$\mathrm{D}=(\mathrm{p}, \mathrm{q}) \mid-\mathrm{m} \leq \mathrm{p} \leq \mathrm{m},-\mathrm{n} \leq \mathrm{q} \leq \mathrm{n},(\mathrm{p}, \mathrm{q}) \neq\left(\begin{array}{ll}0 & 0\end{array}\right)$

$\Theta_{p q}$ is the AR model coefficients and p $\times q$ represents the order of the model. The value of every pixel (say, $y=x i j$ ) is predicted as a linear combination of $D$ neighbouring pixels. That's why in general,

$\mathrm{y}=\mathrm{h} \Theta$

where, $y$ is $\mathrm{P} \times 1$ dimensional, $h$ is a $\mathrm{P} \times \mathrm{D}$ dimensional matrix and $\theta$ is $\mathrm{D} \times 1$ dimensional. Here $y$ records value of each of the $P$ pixels. For each of these $P$ pixels, values of the $D$ neighbouring pixels are recorded in each row of $h$.

\subsection{Estimation of $A R$ coefficients}

Estimation of $\Theta$ can be done by means of bracket notation which is the important part. Let the error in predicting the value of pixel $y$ is denoted by $e$, thus we can write,

$$
\mathrm{e}=\mathrm{y}-\mathrm{h} \Theta
$$
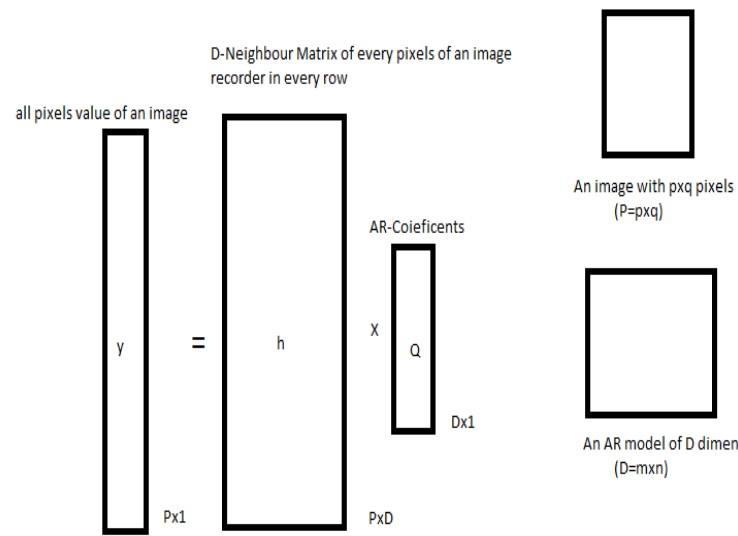

$(P=p \times q)$

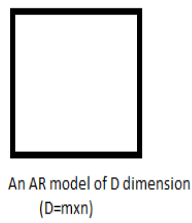

Fig. 1: Different contexts used in estimating AR coefficients

When the AR model is applied to each pixel of an image, it may require large computation for implementation, even if the image is of reasonable size. So, instead of computing AR model for all pixels in binary images, the model in black pixels can be computed only that reduces the requirement of large computation. It should be noted that only about 3\% pixels or less are black in scanned images of hand written text. Regarding the context, here three different templates of neighbouring pixels as shown in fig. 2 denoted by $C 1, C 2$, and $C 3$ as fig. 2(a), (b) and (c), respectively. Lengths of these three contexts are 24,34 , and 34 bits. So, while computing AR model they result in coefficients of 24 order, 34-order, and 34-order AR models.

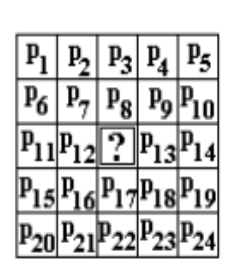

(a)

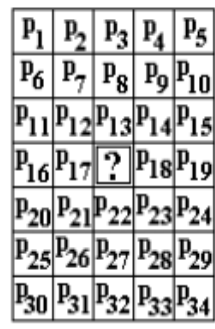

(b) \begin{tabular}{|l|l|l|l|l|l|l|}
\hline $\mathrm{P}_{1}$ & $\mathrm{P}_{2}$ & $\mathrm{P}_{3}$ & $\mathrm{P}_{4}$ & $\mathrm{P}_{5}$ & $\mathrm{P}_{6}$ & $\mathrm{P}_{7}$ \\
\hline & $\mathrm{P}_{8}$ & $\mathrm{P}_{5}$ & & & & \\
\hline
\end{tabular} \begin{tabular}{|l|l|l|l|l|l|l|}
\hline $\mathrm{p}_{8}$ & $\mathrm{p}_{9}$ & $\mathrm{P}_{10}$ & $\mathrm{p}_{11}$ & $\mathrm{p}_{12}$ & $\mathrm{p}_{13}$ & $\mathrm{P}_{14}$ \\
\hline
\end{tabular}

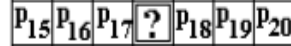

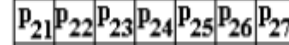
\begin{tabular}{|l|l|l|l|l|l|l|}
\hline $\mathrm{P}_{28}$ & $\mathrm{P}_{29}$ & $\mathrm{p}_{30}$ & $\mathrm{P}_{31}$ & $\mathrm{p}_{32}$ & $\mathrm{P}_{33}$ & $\mathrm{P}_{34}$ \\
\hline
\end{tabular}
Fig. 2: Different contexts used in estimating AR coefficients: (a) $\mathrm{C} 1: 5 \times 5$ context (b) $\mathrm{C} 2: 7 \times 5$ context and (c) C3: $5 \times 7$ context

AR coefficients computed from an image represents its characteristics. We have considered groups, each of them contributes 100 samples in different aspects. Let $\hat{\theta} i$ be the estimated AR model coefficients for the $i$-th image. For an unknown sample, at first the AR model coefficients are computed. Let $\hat{\theta}$ be the estimated coefficients for sample. Euclidean distance between this sample and any of the $N$ samples of the reference database is computed as:

$$
d\left(\hat{\theta}, \hat{\theta}_{i}\right)=\|\hat{\theta}-\hat{\theta} i\|^{2}
$$

\section{B. Methodology based on chain code}

The chain code helps to prevent loss of information and is more useful in image retrieval where the images deal with shapes which are preserved by the contours. Initiating with gray-scale images we have to binarize them using Otsu's global thresholding algorithm. After this component extraction using 8-connectivity can be done. For each of the component, contours are computed, where each contour being a sequence of consecutive boundary points. It is given as:

Contour $_{\mathrm{i}}=\mathrm{pj} \mid \mathrm{j} \leq \mathrm{Mi}, \mathrm{p}_{1}=\mathrm{p}_{\mathrm{mi}}$

where Mi is the length of contour i. Then calculation of the Freeman chain code can be done that is associated with each contour. The sequence is $\left\{c_{j} \mid j \leq M_{i}-1\right\}$ where $\left\{c_{j} \in\right.$ $0,1, \ldots \ldots 7\}$. The original binary image is given by $I$. The boundary pixels are then labelled by their respective codes. After this features extraction can be done from the newly formed image $I^{c}$. Contours are analyzed as local and global levels. At local level, small handwritten fragments are analysed whereas at global level, histograms of chain codes and their variants is employed to remove errors due to a false ordering of the pixels. Finally, the set of extracted histograms is used to characterize an image sample.

\section{EXPERIMENTAL RESULTS}

In this experiment 100 samples of various groups such as humans, flowers, mountains, elephants, monuments, horses, etc. are considered for the comparison purpose from Wang database[9]. This leads to 1000 samples. This way 1000 samples used for training and testing of feed forward back propagation algorithm of ANN Classifier. Training to testing Ratio is maintained 1:1. Preprocessing is done on the samples 
followed by feature extraction. As stated feature extraction is done by both autoregression and chain code methods.

The experimental results for both the feature extraction techniques are given as follows:

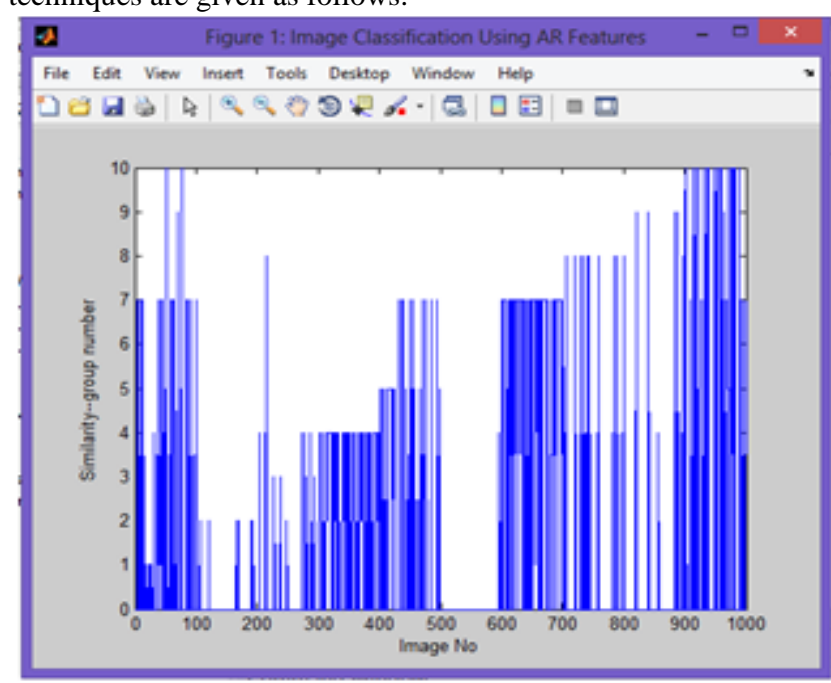

Fig.3- Image classification using AR features

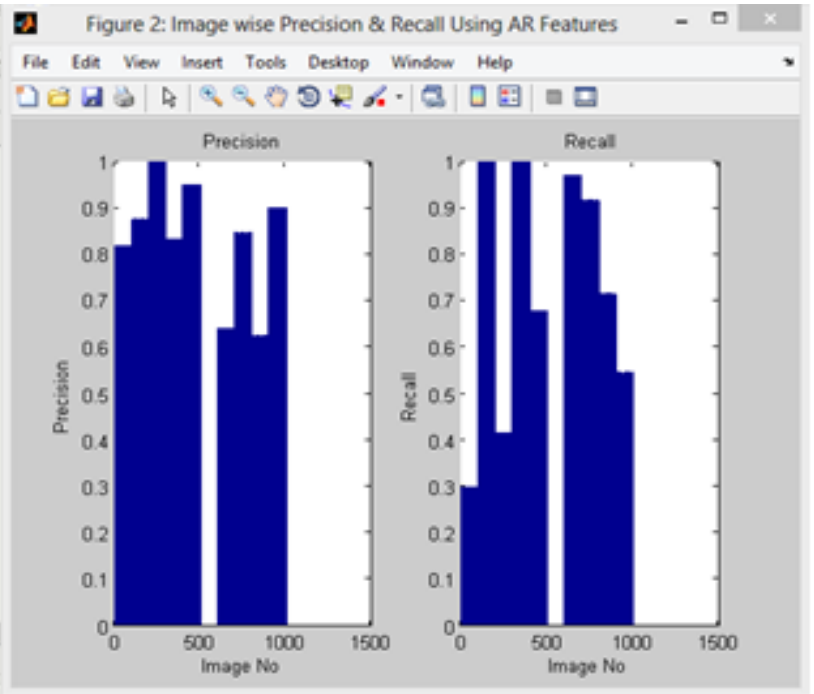

Fig.4- Image showing similarity vs precision and recall for AR features

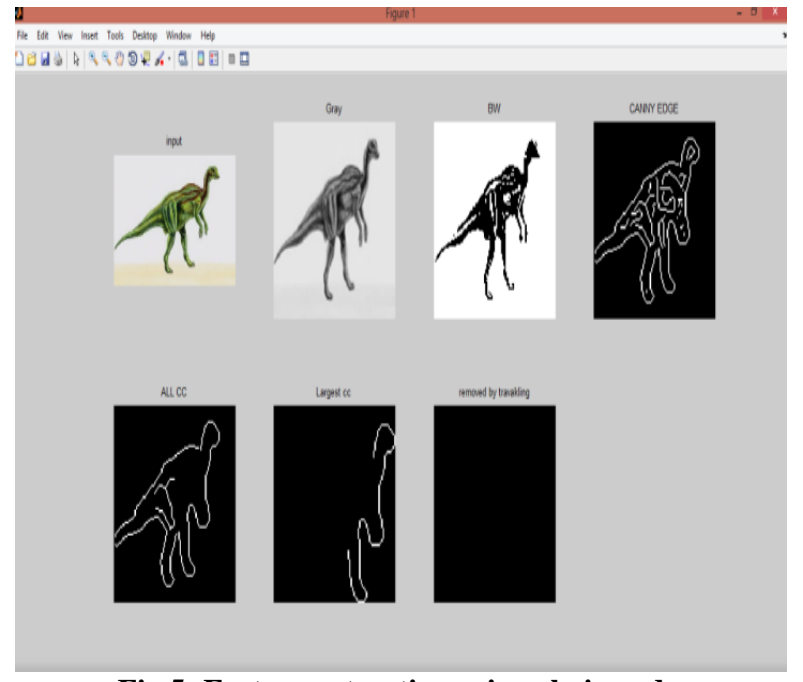

Fig.5- Feature extraction using chain code

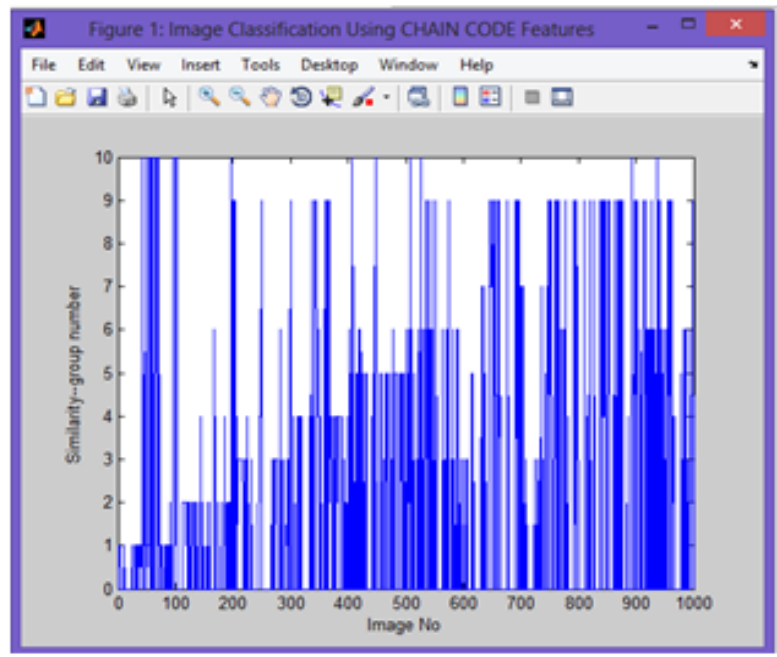

Fig.6- Image classification using chain code features

22 Figure 2: Image wise Precision \& Recall Using CHAIN CODE Featu_. -

File Edin View Inuen Tools Detkeop Window Help

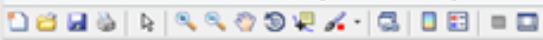

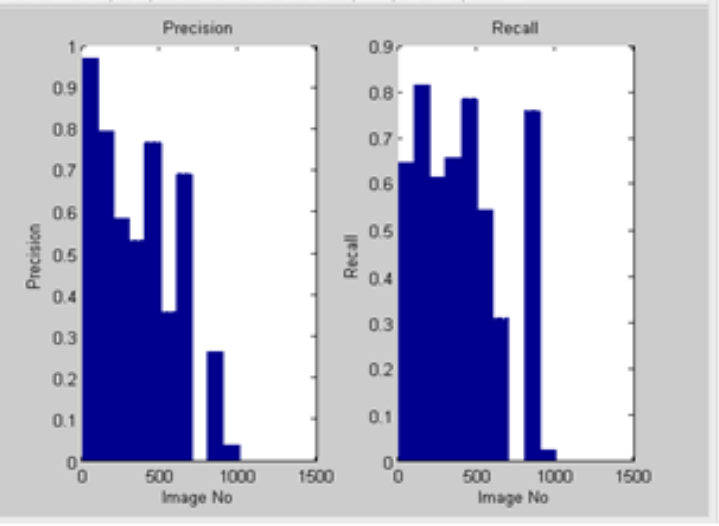

Fig.7- Image showing similarity vs precision and recall for chain code

The experimental results are represented as comparison of both the feature extraction techniques. Table 1 represents the precision and recall on the basis of groups of different clusters. Here the cluster refers to the samples of images which are considered for experimentation purpose. For ex. there are 100 samples of every group such as group of humans, monuments, elephants, flowers, etc. are taken into consideration. Table 2 consist of comparison of performance both the feature extraction techniques i.e. autoregression and chain code, on the basis of precision and recall. Precision and recall can be calculated as:

$$
\begin{gathered}
\text { precision }=\frac{\text { true selected value }}{\text { total selected value }} . . \\
\text { recall }=\frac{\text { true selected value }}{\text { total true value }} . .(8)
\end{gathered}
$$

Table 1- Table showing precision and recall on the basis of groups of different clusters for auto regression

\begin{tabular}{|c|c|c|c|}
\hline Group & Cluster & Precision & Recall \\
\hline Group 1 & Humans & 81.82 & 30 \\
\hline Group 2 & Beach & 87.50 & 100 \\
\hline Group 3 & Monuments & 100 & 41.67 \\
\hline Group 4 & Buses & 83.33 & 100 \\
\hline
\end{tabular}




\begin{tabular}{|c|c|c|c|}
\hline Group 5 & Dinosaurs & 95 & 67.86 \\
\hline Group 6 & Elephants & 0 & 0 \\
\hline Group 7 & Flowers & 64 & 96.97 \\
\hline Group 8 & Horses & 84 & 91.67 \\
\hline Group 9 & Mountains & 62.50 & 71.43 \\
\hline Group 10 & Dishes & 90 & 54.55 \\
\hline
\end{tabular}

Table 2- Table showing precision and recall on the basis of groups of different clusters for chain code

\begin{tabular}{|c|c|c|c|}
\hline Group & Cluster & Precision & Recall \\
\hline Group 1 & Humans & 97.78 & 75.86 \\
\hline Group 2 & Beach & 72.09 & 86.11 \\
\hline Group 3 & Monuments & 67.57 & 58.14 \\
\hline Group 4 & Buses & 56.25 & 65.85 \\
\hline Group 5 & Dinosaurs & 78.26 & 58.06 \\
\hline Group 6 & Elephants & 28 & 41.18 \\
\hline Group 7 & Flowers & 57.14 & 35.29 \\
\hline Group 8 & Horses & 0 & 0 \\
\hline Group 9 & Mountains & 28.43 & 78.38 \\
\hline Group 10 & Dishes & 47.6 & 22.7 \\
\hline
\end{tabular}

Table 3- Table showing maximum precision and recall based on autoregression and chain code

\begin{tabular}{|c|c|c|}
\hline Method & Precision & Recall \\
\hline Autoregression & 90 & 100.00 \\
\hline Chain code & 97.78 & 86.11 \\
\hline
\end{tabular}

\section{CONCLUSIONS}

Based on the previous researches done in the field of image retrieval we came to know that it is somewhat difficult task. Techniques till date are useful but have large computational complexity, some of them are costly. Experimentally both the feature extraction techniques are compared on the basis of precision and recall considering it as performance parameters. The result shows that autoregression technique is reliable and robust than chain code feature extraction for image retrieval.

\section{REFERENCES}

[1] Utpal Garain, Thierry Paquet "Off-Line Multi-Script Writer Identification using AR Coefficients", 10th International Conference on Document Analysis and Recognition, 2009.

[2] A. Bandera, C. Urdiales, F. Arrebola, F. Sandoval, "2D object recognition based on curvature functions obtained from local histograms of the contour chain code”, Pattern Recognition Letters (49 \pm 55$), 1999$.

[3] Imran Siddiqi, Nicole Vincent, "A Set of Chain Code Based Features for Writer Recognition", 10th International Conference on Document Analysis and Recognition,2009.

[4] Kirti Jain, Dr.Sarita Singh Bhadauria, Dr.Gulab Singh,"A Hybrid Technique for Shape Matching Based on chain code and DFS Tree", IOSR Journal of Computer Engineering, Vol. 11, Issue 3, pp 51-56, 2013.

[5] Jongan Park,et.al., "Image Retrieval Technique Using Rearranged Freeman Chain Code", First International Conference on Informatics and Computational Intelligence, 2011.

[6] Farida Khursheed and A.H. Mir, "AR Model Based Human Identification using Ear Biometrics",International Journal of Signal Processing, Image Processing and Pattern Recognition Vol.7, No.3 (2014), pp.347-360.

[7] K. Deguchi, "Two-dimensional Auto-regressive Model for Analysis and Sythesis of Gray-level textures," in Proc. of the 1st Int. Sym. for Science on Form, General Ed. S. Ishizaka, Eds. Y. Kato, R. Takaki, and J. Toriwaki, pp. 441-449, Tokyo, Japan, 1986.

[8] Arvind nagathan, Manimozhi, Jitendranath Mungara ," content-based image retrieval system using feed-forward back propagation neural network " IJCSNS international journal of computer science and network security, vol.14 no.6, June 2014.

[9] Savvash " Benchmark databases for CBIR", 2008 